\title{
A COMPUTER METHOD FOR MOMENT-CURVATURE ANALYSIS OF COMPOSITE STEEL-CONCRETE CROSS-SECTIONS OF ARBITRARY SHAPE
}

\author{
Cosmin G. CHIOREAN \\ Department of Structural Mechanics, Faculty of Civil Engineering, Technical University of Cluj-Napoca, \\ 15 C. Daicoviciu, Str., 400020, Cluj-Napoca, Romania
}

Received 20 October 2016; accepted 09 December 2016

\begin{abstract}
This paper presents a new computer method for moment-curvature analysis of arbitrary-shaped composite steel-concrete cross-sections that are subjected to biaxial bending and axial force. The complete moment-curvature diagrams are determined such that axial force and bending moment ratio is kept constant. A strain-driven algorithm has been developed, the solution of the nonlinear equilibrium equations is controlled by the assumed strain values in the most compressed point and by solving just two coupled nonlinear equations. Such an approach may be used to assess accurately the main features of the elasto-plastic behaviour of composite cross-sections: multiple yielding points associated to different materials, flexural and axial rigidity, moment-curvature relationship in pre and post-critical domain and curvature ductility detecting also unloaded regions of the cross-sections that may occur even under monotonically increasing of the total bending moment. Since the Jacobian's of the resulted nonlinear system of equations is always positive definite the convergence stability is not sensitive to the initial/starting values of the iterative process and to the strain softening exhibited by the concrete in compression. By using a path integral technique on boundary of cross-section area, gradual spread of plasticity and residual stress distribution assumed for encased steel elements are accurately considered reducing also the computational time significantly. In order to illustrate the proposed method and its accuracy and efficiency, a computer program has been developed and used to study several representative examples. The numerical studies presented and comparisons made prove the effectiveness and time saving of the proposed method of analysis.
\end{abstract}

Keywords: moment-curvature analysis, composite steel-concrete sections, biaxial bending, strain softening, residual stresses, path integral approach.

\section{Introduction}

Moment-curvature analysis of cross-sections is of prime importance in the analysis of inelastic behaviour of cross-sections subjected to bi-axial loading and axial force. Such analyses will reveal the strength, ductility, energy dissipation capacity and also the elasto-plastic rigidity of a cross-section section at a specified level of external loading. A complete moment-curvature relationship shows also strength reduction beyond the peak point, when strain-softening exhibited by the concrete is taken into account, and degradation of flexural and axial rigidity of cross-section at different stages of loading and is usually used to determine the interaction diagrams and moment capacity contours of cross-sections (Charalampakis, Koumousis 2008).

Some analytical studies and numerical procedures on the moment-curvature responses of steel and composite (steel-concrete) cross-sections are available in literature. All these methods can be grouped in two basic approaches. The first approach consists of a de-

Corresponding author:

G. Chiorean E-mail: cosmin.chiorean@mecon.utcluj.ro 
formation (i.e. curvature) driven algorithm; in this respect the problem is formulated to evaluate the bending moments corresponding to a given value of the total curvature and a predefined axial force. In these procedures the inclination of neutral axis is kept constant and the unknown represents either the depth of neutral axis or axial strain. These parameters are determined iteratively such that the equilibrium of the axial force to be satisfied (Charalampakis, Koumousis 2008; Marmo et al. 2008; Papanikolau 2012). Such approaches exhibits relatively well numerical stability but the main drawback of this approach is given by the fact that, for arbitrary shape cross-section, the resulted bending moments associated to the prescribed total curvature do not laying in the same plane. In other words, since this approach does not enforce equilibrium for bending moments about each principal axis, spurious bending moments may be introduced and hence inaccuracy in representing in the same plane moment-curvature diagram of the cross-sections with arbitrary shape. The second approach is based upon the explicit solution of the non-linear equilibrium equations for axial force and each bending moment according to the classical Newton's scheme considering the strains as functions of the bending curvatures and axial strain (Rotter 1985; Rodriguez-Gutierrez, Dario Aristizabal-Ochoa 2001). Recently, Chiorean (2014) enhanced this approach by adopting NewtonRaphson iterative procedure in conjunction with the arc-length constrain equation controlling in this way the nonlinear solution both in forces and deformations and solving in this way the three coupled equilibrium equations. However all these approaches have been developed only for monotonically loading and the main limitation consists in the lack of potentials to be extended to the more complex analyses such as cyclic moment-curvature analysis.

A new incremental-iterative procedure is developed here to determine the complete moment-curvature response of arbitrary-shaped composite steelconcrete cross-sections. The diagrams are determined such that axial force and bending moment ratio is kept constant for a prescribed value of the strain in the most compressed point of the concrete matrix. In the proposed approach the three coupled nonlinear equilibrium equations are manipulated so that one of them is uncoupled and the Newton iterative strategy is applied only to the remaining coupled equilibrium equations. Such an approach is advantageous with respect to the existing ones, in that the solution is obtained by solv- ing just two coupled nonlinear equations. Moreover, since the Jacobian's of the resulted nonlinear system of equations is always positive definite the convergence stability is not sensitive to the initial/starting values of the iterative process and to the strain softening exhibited by the concrete in compression. Since the solution is controlled by predefined values of strains in most compressed concrete fiber, this approach seems to be effective to be extended to the more complex cyclic moment-curvature analysis, by continuously varying (loading/unloading) the extreme concrete fiber strain; in this way the complete moment-curvature diagram for given bending moment's ratio under a prescribed axial force can be revealed. However, the stress-path dependency of concrete and steel is not taken into account in the proposed approach; the elastic unloading of yielded fibers is ignored in this study. The proposed method addresses computational efficiency and modelling shortcomings also through the use of path integral approach for transforming stress resultant and stiffness coefficients (i.e. Jacobian's coefficients) into line integrals and then performing in this way numerical integration of the cross-sectional nonlinear characteristics and residual stresses, enabling in this way accurate geometrical specifications and precise modelling of arbitrary shaped cross-sections. However, it is important to underline that when either cyclic analysis or elastic unloading effect is taken into account either fiber-decomposition of cross-section or fiber-free approaches (Marmo 2007) have to be considered in the proposed approach.

In order to validate the effectiveness of the numerical method developed here several computational examples are given and discussed. From the extensive numerical studies, some of them presented here, the proposed approach has been found to be very effective to determine the complete moment-curvature relationships showing strength reduction beyond the multiple peak points and degradation of flexural and axial rigidity of cross-section at different stages of loading.

\section{Mathematical formulation}

\subsection{Basic assumptions and constitutive material models}

Consider the cross-section subjected to the action of the external bending moments about both global axes and axial force as shown in Figure 1. The cross-section may assume any shape with multiple polygonal or circular openings. It is assumed that plane section remains plane after deformation. 


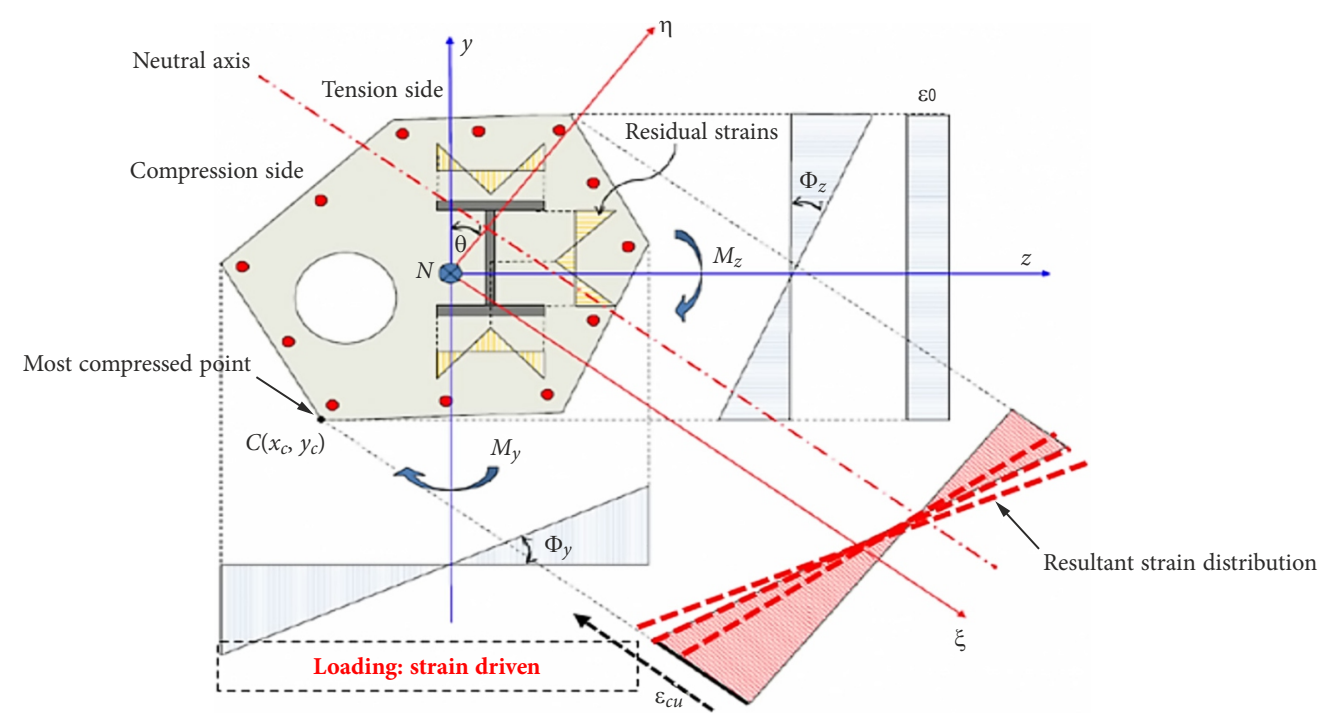

Fig. 1. Model of arbitrary composite cross-section. Strain-driven approach

This implies a full interaction between the steel and concrete components of a composite concretesteel cross section. Shear and torsional interaction effects are not accounted for, neither in the concrete nor in the steel constitutive models. Thus the resultant strain distribution corresponding to the curvatures about each global axes $\Phi=\left\{\phi_{z}, \phi_{y}\right\}$ and the axial compressive strain $\varepsilon_{0}$ can be expressed at a generic point, in concrete matrix fiber and structural steel or ordinary reinforced bars, of coordinates $(z, y)$ in a linear form as:

$$
\varepsilon \varepsilon_{0}, \phi_{z}, \phi_{y}=\varepsilon_{0}+\phi_{z} y+\phi_{y} z .
$$

The stress-path dependency of concrete and steel is not taken into account in the proposed approach. Hence, in the proposed approach, the elastic unloading of yielded fibers will be ignored. The nonlinear stress-strain relationships to model the concrete under compression is represented by a combination of a second-degree parabola (for ascending part) and a straight line (for descending part), Eq. (2), as depicted in Figure 2a:

$$
f_{c}=\left\{\begin{array}{l}
f_{c}^{\prime \prime}\left(2 \frac{\varepsilon}{\varepsilon_{c 0}}-\frac{\varepsilon^{2}}{\varepsilon_{c 0}^{2}}\right), \quad \varepsilon \leq \varepsilon_{0} \\
f_{c}^{\prime \prime}\left(1-\gamma\left(\frac{\varepsilon-\varepsilon_{c 0}}{\varepsilon_{c u}-\varepsilon_{c 0}}\right)\right), \varepsilon_{0}<\varepsilon
\end{array},\right.
$$

where $\gamma$ represents the degree of confinement in the concrete and allows for the modelling of strain-softening, creep and confinement in concrete by simply varying the crushing strain $\varepsilon_{c 0}$, ultimate compressive strain $\varepsilon_{c u}$ and $\gamma$ respectively (Chiorean 2014). The model to account for tension stiffening developed by Vecchio and Collins (Vecchio, Collins 1986) or that given in CEB-FIP (1993) may be included in the proposed method but this effect is ignored in the present investigation. A multi-linear elastic-plastic stress-strain relationship, both in tension and in compression, is assumed for the structural steel and the conventional reinforcing bars. In this way the strain-hardening effect may be included in analysis. The analytical model

a)
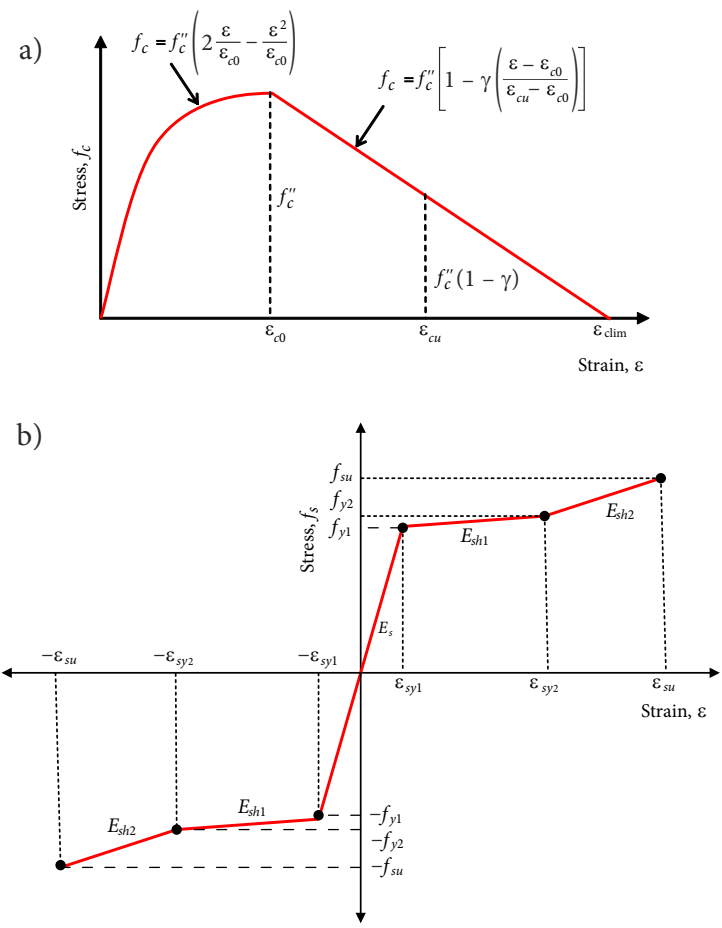

Fig. 2. Stress-strain relationships for (a) concrete in compression and (b) steel 
can be given in the following form (Chiorean 2013) (Fig. 2b):

$f_{s}=\left\{\begin{array}{l}E_{s} \varepsilon,|\varepsilon| \leq\left|\varepsilon_{s y 1}\right| \\ \operatorname{sgn}(\varepsilon) f_{y 1}+E_{s h 1}\left(\varepsilon-\operatorname{sgn}(\varepsilon) \cdot \varepsilon_{s y 1}\right), \varepsilon_{s y 1}<|\varepsilon| \leq \varepsilon_{s y 2}, \\ \operatorname{sgn}(\varepsilon) f_{y 2}+E_{s h 2}\left(\varepsilon-\operatorname{sgn}(\varepsilon) \cdot \varepsilon_{s y 2}\right), \varepsilon_{s y 2}<|\varepsilon| \leq \varepsilon_{s u}\end{array}\right.$

where $E_{s}$ is the Young modulus, $f_{s y i}$ denotes the yield stresses, $\varepsilon_{s y i}$ represents the yield strains, $\varepsilon_{s u}$ the ultimate steel strain and $E_{s h i}$ represents the slopes of the yielding branch.

Residual stresses for encased steel profile may be incorporated in the analysis. The magnitude and distribution of these initial stresses in hot-rolled members shows complex shapes and depends on the type of cross section and manufacturing process and hence the assumed distribution and the magnitude represents usually only a modelling convenience. The presence of residual stresses in conjunction with high values of axial loads affects the spread of plasticity throughout the height of the steel section and hence may significantly influence both the ultimate strength capacity and inelastic deformability of the composite steel-concrete cross-section. The distribution of residual stresses is self-equilibrated (i.e. internal resultant efforts are equal with zero) and different patterns are proposed in literature, some of them are depicted in Figure 3 for $\mathrm{H}$ sections. In the US, for $\mathrm{H}$-sections the residual stress is considered constant in the web although, when the depth of a wide flange section is large, it varies more or less parabolically (Fig. 3b) (Chiorean 2017). Another possible residual stress pattern in the web is the one simplified by a linear variation as used in European calibration frames (Fig. 3a). When the residual stresses are taken into account in the inelastic analysis the total strain in a current point of a structural steel can be computed as:

$$
\varepsilon\left(\varepsilon_{0}, \phi_{z}, \phi_{y}\right)=\varepsilon_{0}+\phi_{z} y+\phi_{y} z+\varepsilon_{r},
$$

where $\varepsilon_{r}\left(=\sigma_{r} / E\right)$ represents the residual strain at that point, $\sigma_{r}$ denotes the residual stress and $E$ represents the Young modulus.

\subsection{Formulation of the proposed method}

In order to determine the moment curvature diagrams of composite steel concrete cross-sections subjected to axial force and given bending moment ratio $\left(\tan (\alpha)=M_{y} / M_{z}\right)$ a new incremental-iterative procedure is proposed. A strain-driven algorithm is developed and the full moment-curvature diagram is computed by continuously increasing the strain in most compressed concrete fibre of cross-section. Consider a composite cross-section with arbitrary shape as shown in Figure 1 subjected to biaxial bending moments $\left(M_{z 0}, M_{y 0}\right)$ and axial force $\left(N_{0}\right)$. The global $z, y$-axes of the cross section could have arbitrary origin but usually it is assumed to be placed either in the elastic or plastic centroid of the cross-section. The equilibrium is achieved when the external forces $\left(N_{0}, M_{z 0}\right.$, and $\left.M_{y 0}\right)$ are equal to the internal ones. These conditions can be represented mathematically in terms of the following nonlinear system of equations as:

$$
\left\{\begin{array}{l}
\int_{A_{c s}} \sigma\left(\varepsilon\left(\varepsilon_{0}, \phi_{z}, \phi_{y}\right)\right) d A_{c s}+\sum_{i=1}^{N_{r s}} \sigma\left(\varepsilon_{i}\left(\varepsilon_{0}, \phi_{z}, \phi_{y}\right)\right) A_{r s i}-N_{0}=0 \\
\int_{A_{c s}} \sigma\left(\varepsilon\left(\varepsilon_{0}, \phi_{z}, \phi_{y}\right)\right) y d A_{c s}+\sum_{i=1}^{N_{r s}} \sigma\left(\varepsilon_{i}\left(\varepsilon_{0}, \phi_{z}, \phi_{y}\right)\right) y_{i} A_{r s i}-M_{z 0}=0, \\
\int_{A_{c s}} \sigma\left(\varepsilon\left(\varepsilon_{0}, \phi_{z}, \phi_{y}\right)\right) x d A_{c s}+\sum_{i=1}^{N_{r s}} \sigma\left(\varepsilon_{i}\left(\varepsilon_{0}, \phi_{z}, \phi_{y}\right)\right) z_{i} A_{r s i}-M_{y 0}=0
\end{array}\right.
$$

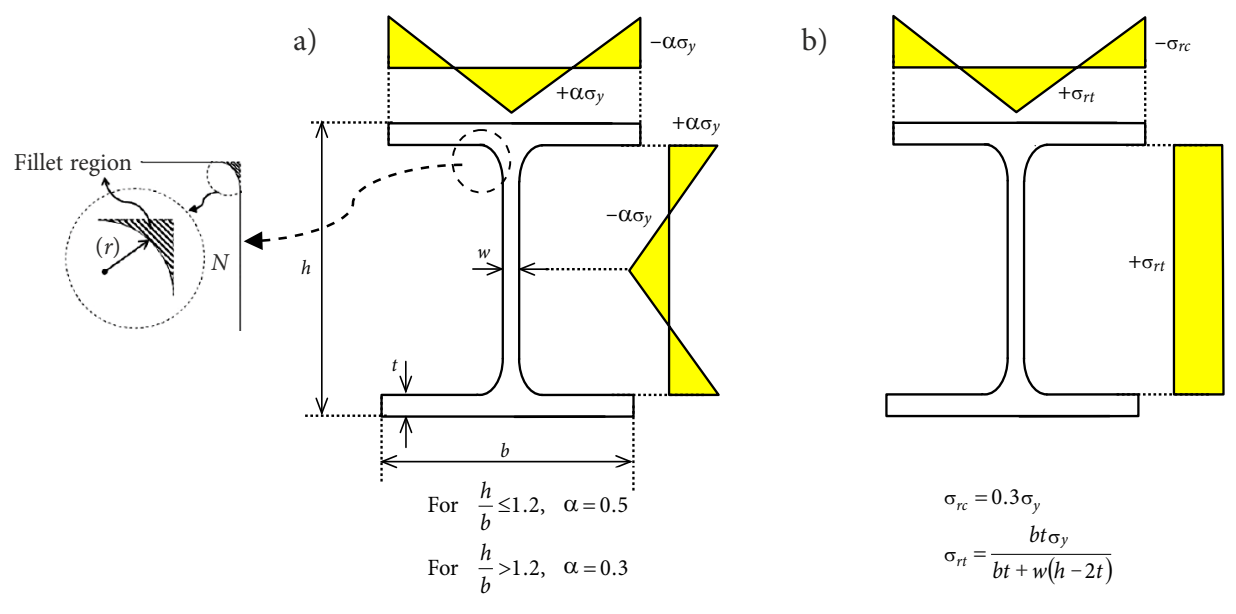

Fig. 3. Residual stress $\left(\sigma_{r}\right)$ patterns and fillet regions of steel sections 
where $A_{r s i}\left(i=1, N_{r s}\right)$ denotes the resisting cross-section area of the $i$-th bar located at $\left(z_{i}, y_{i}\right)$ coordinates about the reference system $(z O y), N_{r s}$ denotes the total number of conventional steel reinforcements, the surface integral is extended over steel and concrete areas $\left(A_{c s}\right)$, and in which $\varepsilon_{0}, \phi_{z}, \phi_{y}$ represent the unknowns. One point on the moment curvature diagram correspond to a prescribed value of the concrete compressive strain at the outer compressed point of the concrete section (i.e. $\varepsilon_{c, \max }$ equal to the prescribed value of compressive strain $\varepsilon_{c}$ ). Considering the irregular composite section as shown in Figure 1, for each orientation of the neutral axis defined by the curvatures $\phi_{z}$ and $\phi_{y}$ ( $\left.\operatorname{tg} \theta=\phi_{y} / \phi_{z}\right)$ the farthest point, with the co-ordinates $x_{c}, y_{c}$, on the compression side is determined. Assuming the prescribed value $\varepsilon_{c}$ for the strain at this point:

$$
\varepsilon_{0}+\phi_{z} y_{c}+\phi_{y} z_{c}=\varepsilon_{c}
$$

the axial strain $\varepsilon_{0}$ can be expressed as:

$$
\varepsilon_{0}=\varepsilon_{c}-\left(\phi_{z} y_{c}+\phi_{y} z_{c}\right)
$$

and then by combining the Eq. (1) and Eq. (7), the resulting strain distribution corresponding to the curvatures $\phi_{z}$ and $\phi_{y}$ can be expressed in linear form as:

$$
\tilde{\varepsilon}\left(\phi_{z}, \phi_{y}\right)=\varepsilon_{c}+\phi_{z} y-y_{c}+\phi_{y}\left(z-z_{c}\right) .
$$

In this way, the basic equations of equilibrium (5) can be decoupled and written in the following form:

$$
\left\{\begin{array}{l}
\int_{A_{c s}} \sigma\left(\tilde{\varepsilon}\left(\phi_{z}, \phi_{y}\right)\right) d A_{c s}+\sum_{i=1}^{N_{r s}} \sigma\left(\tilde{\varepsilon}_{i}\left(\phi_{z}, \phi_{y}\right)\right) A_{r s i}-N_{0}=0 \\
\int_{A_{c s}} \sigma\left(\tilde{\varepsilon}\left(\phi_{z}, \phi_{y}\right)\right) z d A_{c s}+\sum_{i=1}^{N_{r s}} \sigma\left(\tilde{\varepsilon}_{i}\left(\phi_{z}, \phi_{y}\right)\right) z_{i} A_{r s i}- \\
\tan \alpha\left[\int_{A_{c s}} \sigma\left(\tilde{\varepsilon}\left(\phi_{z}, \phi_{y}\right)\right) y d A_{c s}+\sum_{i=1}^{N_{r s}} \sigma\left(\tilde{\varepsilon}_{i}\left(\phi_{z}, \phi_{y}\right)\right) y_{i} A_{r s i}\right]=0 \\
\int_{A_{c s}} \sigma\left(\tilde{\varepsilon}\left(\phi_{z}, \phi_{y}\right)\right) y d A_{c s}+\sum_{i=1}^{N_{r s}} \sigma\left(\tilde{\varepsilon}_{i}\left(\phi_{z}, \phi_{y}\right)\right) y_{i} A_{r s i}-M_{z}=0
\end{array}\right.
$$

in which the bending moment about $z$-axis $M_{z}$ and curvatures $\phi_{z}$ and $\phi_{y}$ becomes the main unknowns. Regarding the curvatures $\phi_{z}$ and $\phi_{y}$ as independent variables in the third equation (i.e. bending moment equation), then

$$
M_{z}=\int_{A_{c s}} \sigma\left(\tilde{\varepsilon}\left(\phi_{z}, \phi_{y}\right)\right) y d A_{c s}+\sum_{i=1}^{N_{r s}} \sigma\left(\tilde{\varepsilon}_{i}\left(\phi_{z}, \phi_{y}\right)\right) y_{i} A_{r s i}
$$

and the third equation from the above three coupled nonlinear equations can be eliminated and the curva- tures $\phi_{z}$ and $\phi_{y}$ can be determined by solving just the following two coupled nonlinear equations:

$$
\begin{aligned}
& \mathbf{F}(\boldsymbol{\Phi})=\mathbf{0} ; \\
& \mathbf{F}=\left[f_{1}\left(\phi_{z}, \phi_{y}\right), f_{2}\left(\phi_{z}, \phi_{y}\right)\right]^{T} ; \boldsymbol{\Phi}=\left[\phi_{z}, \phi_{y}\right]^{T},
\end{aligned}
$$

where:

$$
\left\{\begin{array}{l}
f_{1}\left(\phi_{z}, \phi_{y}\right)=\int_{A_{c s}} \sigma\left(\tilde{\varepsilon}\left(\phi_{z}, \phi_{y}\right)\right) d A_{c s}+\sum_{i=1}^{N_{r s}} \sigma\left(\tilde{\varepsilon}_{i}\left(\phi_{z}, \phi_{y}\right)\right) A_{r s i}-N_{0}=0 \\
f_{2}\left(\phi_{z}, \phi_{y}\right)=\int_{A_{c s}} \sigma\left(\tilde{\varepsilon}\left(\phi_{z}, \phi_{y}\right)\right) z d A_{c s}+\sum_{i=1}^{N_{r s}} \sigma\left(\tilde{\varepsilon}_{i}\left(\phi_{z}, \phi_{y}\right)\right) z_{i} A_{r s i}- \\
\tan \alpha\left[\int_{A_{c s}} \sigma\left(\tilde{\varepsilon}\left(\phi_{z}, \phi_{y}\right)\right) y d A_{c s}+\sum_{i=1}^{N_{r s}} \sigma\left(\tilde{\varepsilon}_{i}\left(\phi_{z}, \phi_{y}\right)\right) y_{i} A_{r s i}\right]=0
\end{array} .\right.
$$

Hence, for a given value of axial force $N_{0}$ and a given bending moment ratio $\tan (\alpha)=M_{y} / M_{z}$, one may solve for $\phi_{z}$ and $\phi_{y}$ the nonlinear system (12) and then the bending moment $M_{z}$ can be simply evaluated using Eq. (10) and then $M_{y}=\tan (\alpha) M_{z}$. According to the Newton iterative method, the iterative changes of unknowns' vector $\Phi$ can be written as:

$$
\boldsymbol{\Phi}^{(i+1)}=\boldsymbol{\Phi}^{(i)}-\mathbf{K}_{T}^{-1}\left(\boldsymbol{\Phi}^{(i)}\right) \cdot \mathbf{F}\left(\boldsymbol{\Phi}^{(i)}\right), \quad i \geq 0 .
$$

The iterative procedure starts with the initial values of the curvatures $\phi_{z}=\phi_{z 0}$ and $\phi_{y}=\phi_{y 0}(k=0)$, specifying the axial force $N_{0}$ and the angle $\alpha$, formed by the bending moments $M_{z}$ and $M_{y}$. For the next iterations an adaptive-descent algorithm is applied (Chiorean 2010). In this way, for a given value of axial force $N_{0}$ and a given bending moment ratio $\tan (\alpha)=M_{y} / M_{z}$ the moment-curvature diagram can be directly determined, by continuously varying the strain in most compressed point with increments $\Delta \varepsilon_{\mathrm{c}}$ :

$$
\varepsilon_{c, k}=\varepsilon_{c, k-1}+\Delta \varepsilon_{c} \leq \varepsilon_{c, \lim }, k=1,2, \ldots
$$

where $\varepsilon_{c, 0}$ represents the initial axial strain associated to the axial force $N_{0}$ applied together with small bending moments and $\varepsilon_{c \text {,lim }}$ represents the limit compression strain (Fig. 2a). The iteration procedure is conducted with the tangent Jacobian's matrix coefficients computed as:

$$
\mathbf{K}_{T}=\left[\begin{array}{ll}
\frac{\partial f_{1}}{\partial \phi_{x}} & \frac{\partial f_{1}}{\partial \phi_{y}} \\
\frac{\partial f_{2}}{\partial \phi_{x}} & \frac{\partial f_{2}}{\partial \phi_{x}}
\end{array}\right]=\left[\begin{array}{ll}
k_{11} & k_{12} \\
k_{21} & k_{22}
\end{array}\right],
$$

where the matrix coefficients are detailed in the following: 


$$
\begin{aligned}
& k_{11}=\frac{\partial f_{1}}{\partial \phi_{z}}=\int_{A_{c s}} \frac{\partial \sigma}{\partial \tilde{\varepsilon}} \frac{\partial \tilde{\varepsilon}}{\partial \phi_{z}} d A+\sum_{i=1}^{N_{r s}} \frac{\partial \sigma_{i}}{\partial \varepsilon_{i}} \frac{\partial \tilde{\varepsilon}_{i}}{\partial \phi_{z}} A_{r s i}= \\
& \int_{A_{c s}} E_{T}\left(y-y_{c}\right) d A+\sum_{i=1}^{N_{r s}} E_{T i}\left(y_{i}-y_{c}\right) A_{r s i} ; \\
& k_{12}=\frac{\partial f_{1}}{\partial \phi_{y}}=\int_{A_{c s}} \frac{\partial \sigma}{\partial \tilde{\varepsilon}} \frac{\partial \tilde{\varepsilon}}{\partial \phi_{y}} d A+\sum_{i=1}^{N_{r s}} \frac{\partial \sigma_{i}}{\partial \tilde{\varepsilon}_{i}} \frac{\partial \tilde{\varepsilon}_{i}}{\partial \phi_{y}} A_{r s i}= \\
& \int_{A_{c s}} E_{T}\left(z-z_{c}\right) d A+\sum_{i=1}^{N_{r s}} E_{T i}\left(z_{i}-z_{c}\right) A_{r s i} ; \\
& k_{21}=\frac{\partial f_{2}}{\partial \phi_{z}}=\int_{A_{c s}} \frac{\partial \sigma}{\partial \tilde{\varepsilon}} \frac{\partial \tilde{\varepsilon}}{\partial \phi_{z}} z d A+\sum_{i=1}^{N_{r s}} \frac{\partial \sigma_{i}}{\partial \tilde{\varepsilon}_{i}} \frac{\partial \tilde{\varepsilon}_{i}}{\partial \phi_{z}} A_{r s i}- \\
& \tan (\alpha)\left[\int_{A_{c s}} \frac{\partial \sigma}{\partial \tilde{\varepsilon}} \frac{\partial \tilde{\varepsilon}}{\partial \phi_{z}} y d A+\sum_{i=1}^{N_{r s}} \frac{\partial \sigma_{i}}{\partial \tilde{\varepsilon}_{i}} \frac{\partial \tilde{\varepsilon}_{i}}{\partial \phi_{z}} y_{i} A_{r s i}\right]= \\
& \int_{A_{c s}} E_{T}\left(y-y_{c}\right) z d A+\sum_{i=1}^{N_{r s}} E_{T i}\left(y_{i}-y_{c}\right) z_{i} A_{r s i}- \\
& \tan (\alpha)\left[\int_{A_{c s}} E_{T}\left(y-y_{c}\right) y d A+\sum_{i=1}^{N_{r s}} E_{T i}\left(y_{i}-y_{c}\right) y_{i} A_{r s i}\right] ; \\
& \int_{A_{c s}} E_{T}\left(z-z_{c}\right) z d A+\sum_{i=1}^{N_{r s}} E_{T i}\left(z_{i}-z_{c}\right) z_{i} A_{r s i}- \\
& \tan (\alpha)\left[\int_{A_{c s}} E_{T}\left(z-z_{c}\right) y d A+\sum_{i=1}^{N_{r s}} E_{T i}\left(z_{i}-z_{c}\right) y_{i} A_{r s i}\right] \\
& k_{22}=\frac{\partial f_{2}}{\partial \phi_{y}}=\int_{A_{c s}} \frac{\partial \sigma}{\partial \tilde{\varepsilon}} \frac{\partial \tilde{\varepsilon}}{\partial \phi_{y}} z d A+\sum_{i=1}^{N_{r s}} \frac{\partial \sigma_{i}}{\partial \tilde{\varepsilon}_{i}} \frac{\partial \tilde{\varepsilon}_{i}}{\partial \phi_{y}} z_{i} A_{r s i}- \\
& {\left[\phi_{y} \frac{\partial \sigma}{\partial \tilde{\varepsilon}} y d A+\sum_{r s}^{N_{s}} \frac{\partial \sigma_{i}}{\partial \tilde{\varepsilon}_{i}} \frac{\partial \tilde{\varepsilon}_{i}}{\partial \phi_{y}} y_{r} A_{r s i}\right]=}
\end{aligned}
$$

in which the partial derivatives are expressed with respect to the strains and stresses evaluated at current iteration and in terms of tangent modulus of elasticity $E_{t}$.

In this way, by varying the extreme concrete fiber strain between $\varepsilon_{c, 0}$ and $\varepsilon_{c, \text { lim }}$ (i.e. $\varepsilon_{c, 0} \leq \varepsilon_{c} \leq \varepsilon_{c, \text { lim }}$ ) we can draw the complete moment-curvature diagram for a given bending moment's ratio $(\tan (\alpha))$ and given axial force. The convergence criterion is expressed as an absolute value of the difference between two consecutive approximations as:

$$
\left\|\Phi^{(i+) 1}-\Phi^{(i)}\right\|<t o l,
$$

where $t o l$ is the specified computational tolerance, usually taken as $1 E-10$.

In order to determine the most compressed or tensioned point (fibre) of the cross-section associated to a given value of axial force and bending moment ratio, before to start the proposed approach the crosssection is analyzed considering the section subjected to axial force $N_{0}$ and small values for the bending moments about each principal axes $\left(M_{z 0}, M_{y 0}\right)$. In this respect the system (5) is solved by applying the iterative Newton method as described in (Chiorean 2013). In this way the control point with coordinates $\left(x_{c}, y_{c}\right)$ and initial value for the reference strain can be obtained.

Figure 4 shows a simplified flowchart of the procedure developed for moment-curvature analysis of arbitrary shaped composite steel-concrete cross-sections subjected to axial force and bi-axial bending moments.

\subsection{Evaluation of internal resultant efforts and cross-section tangent stiffness matrix coefficients}

In the proposed approach the boundary integration method based on Green's theorem for arbitrary 2D domains is adopted for evaluation of resultant efforts and Jacobian's coefficients of the nonlinear system (12). In this specific case, this theorem can be stated as:

$$
\begin{aligned}
& \iint_{A}\left[\frac{\partial g(\xi, \eta)}{\partial \xi}-\frac{\partial f(\xi, \eta)}{\partial \eta}\right] d \xi d \eta= \\
& \oint_{C}[f(\xi, \eta) d \xi+g(\xi, \eta) d \eta]
\end{aligned}
$$

where $C$ denotes a positively oriented (counterclockwise), piecewise-smooth, simple closed curve in the plane and $A$ represents the region bounded by $C, g$ and $f$ are functions with continuous partial derivatives on an open region that contains $A$. The internal resultant efforts and Jacobian's coefficients can be expressed by area integrals in a general form as (Chiorean 2017):

$$
I=\iint_{A} \sigma^{(r)}[\varepsilon(z, y)] z^{m} y^{n} d z d y,
$$

where $(r=0,1)$ denotes the order of derivative of the stress $\sigma$ in respect with strain $\varepsilon$, i.e. $r=0$ for resultant efforts, $r=1$ for tangent stiffness coefficients and $(m, n)$ are exponents depending on the requested resultant effort and tangent stiffness coefficient respectively, i.e. $(m, n)=(0,0)$ for resultant axial force; $(0,1)$ for resultant bending moment in respect with $z$-axis; $(1,0)$ for resultant bending moment in respect with $y$-axis; $(0,0)$ for tangent axial rigidity; $(0,2)$ for the second moment of the tangent axial rigidity in respect with $z$-axis, etc.

In order to apply this path integral approach for evaluation of the surface integral (19), the stress field throughout the cross-section is expressed as a single 


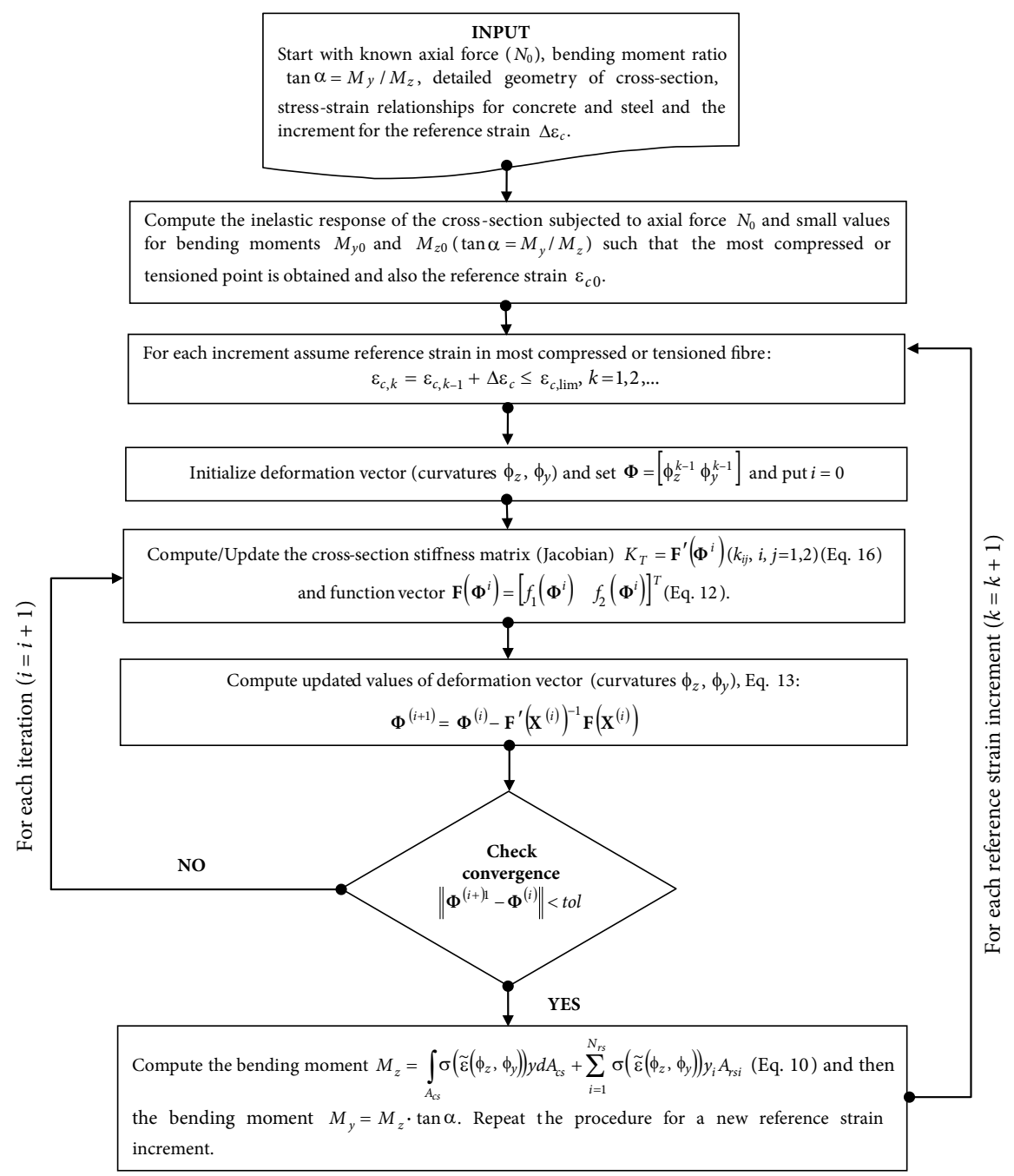

Fig. 4. Simplified analysis flowchart of the proposed procedure

variable function. In this respect the variables $(z, y)$ are transformed by rotating the reference axes $z, y$ to $\xi$, poriented parallel to a uniform pattern given by the angle $\theta$ (Fig. 1):

$$
\left\{\begin{array}{l}
z=\varphi_{1}(\xi, \eta)=\xi \cos \theta+\eta \sin \theta \\
y=\varphi_{2}(\xi, \eta)=-\xi \sin \theta+\eta \cos \theta
\end{array} .\right.
$$

For instance, for cross-sections that admit a single neutral axis ( $\left.\operatorname{tg} \theta=\frac{\phi_{y}}{\phi_{z}}\right)$, the stress field can be enforced to a uniform pattern in the direction given by the current orientation of the neutral axis (Rotter 1985; Bonet et al. 2004; Chiorean 2010) and consequently the strains, stresses and tangent modulus of elasticity can be expressed as a function of the single variable ( $\eta$ ) as:

$$
\begin{gathered}
\varepsilon(z, y) \rightarrow \varepsilon(\eta)=\varepsilon_{0}+\phi \eta ; \\
\sigma[\varepsilon(z, y)] \rightarrow \sigma[\varepsilon(\eta)]
\end{gathered}
$$

$E_{T}(z, y)=\frac{\partial \sigma[\varepsilon(z, y)]}{\partial \varepsilon} \rightarrow E_{T}(\eta)=\frac{\partial \sigma[\varepsilon(\eta)]}{\partial \varepsilon}$

where $\phi$ represents the total curvature $\phi=\sqrt{\phi_{z}^{2}+\phi_{y}^{2}}$. Such situations are encontered for the most cases of composite sections when the perfect bond hypothesis at the interfaces of different components (i.e. concrete and steel) is assumed. Based on this transformation the surface integral given in Eq. (19) can be further expressed as (Chiorean 2017):

$$
\begin{aligned}
& \iint_{A} \sigma^{(r)}[\varepsilon(z, y)] z^{m} y^{n} d z d y= \\
& \iint_{A} \sigma^{(r)}[\varepsilon(\eta)] \varphi_{1}(\xi, \eta)^{m} \varphi_{2}(\xi, \eta)^{n}|J| d \xi d \eta,
\end{aligned}
$$

where $|J|=1$ represents the determinant of the transformation Jacobian given by the Eqs (16). Now with $f=0$ and $g(\xi, \eta)=\sigma^{(r)}[\varepsilon(\eta)] \mathrm{H}(\xi, \eta)$ the formula (18) can be rewritten as: 


$$
\begin{aligned}
& \iint_{A} \sigma^{(r)}[\varepsilon(\eta)] \varphi_{1}(\xi, \eta)^{m} \varphi_{2}(\xi, \eta)^{n} d \xi d \eta= \\
& \oint_{C} \sigma^{(r)}[\varepsilon(\eta)] H(\xi, \eta) d \eta
\end{aligned}
$$

where

$$
\mathrm{H}(\xi, \eta)=\int \varphi_{1}(\xi, \eta)^{m} \varphi_{2}(\xi, \eta)^{n} d \xi
$$

and in this case $A$ represents the surface and $C$ is positivey oriented boundary of the entire cross-section, respectively.

It is worth noticing that when either the partial composite action (i.e. partial shear connection) or initial stresses (i.e. residual stresses for steel component) with non-uniform distribution have to be taken into account the neutral axis is no longer unique for the whole cross-section. Consequently such a simple transformation can not be performed for the whole composite cross-section. Such situations occurs for composite steel-concrete cross-sections when the effect of initial residual stresses is taken into account for the encased steel profile. However, the effect of residual stresses may be included in this approach providing that initial stresses can be linearized for individual zones associated to different distributions of these stresses throughout the height of cross-section. For instance assuming the EC3 distribution of residual stresses the steel cross-section conceptually has to be divided in six regions as depicted in Figure 3a, according with changes in the residual stress pattern. In this way the total strain can be expressed as in Eq. (4) for each re- gion, where $\varepsilon_{r}$ represents a linear residual strain field which can be expressed for each particularly region $(i)$ as (Chiorean 2017):

$$
\varepsilon_{r}^{(i)}(z, y)=a_{1}^{(i)}+a_{2}^{(i)} z+a_{3}^{(i)} y .
$$

Thus the total strain in a current point $(z, y)$ of a particular steel region $(i)$ can be expressed as:

$$
\varepsilon^{(i)}(z, y)=\left(\varepsilon_{0}+a_{1}^{(i)}\right)+\left(\phi_{y}+a_{2}^{(i)}\right) z+\left(\phi_{z}+a_{3}^{(i)}\right) y .
$$

Next, the integration of the stress resultant and Jacobian's coefficients over the steel cross-section will be transformed into line integrals along the perimeter of each individual regions in which the steel cross-section has been decomposed, but in this case the reference axes are rotated for each steel region using the following value for angle $\theta^{(i)}$ (Chiorean 2017), (Fig. 5a):

$$
\tan \theta^{(i)}=\frac{\phi_{y}+a_{2}^{(i)}}{\phi_{z}+a_{3}^{(i)}}
$$

and:

$$
\begin{aligned}
& \varepsilon^{(i)}(z, y) \rightarrow \varepsilon\left(\eta^{(i)}\right)=\varepsilon_{0}+a_{1}^{(i)}+\phi^{(i)} \eta^{(i)} ; \varphi^{(i)}= \\
& \sqrt{\left(\phi_{z}+a_{3}^{(i)}\right)^{2}+\left(\phi_{y}+a_{2}^{(i)}\right)^{2}} ; \\
& \sigma\left[\varepsilon^{(i)}(z, y)\right] \rightarrow \sigma\left[\varepsilon\left(\eta^{(i)}\right)\right] ; \\
& E_{T}(z, y)=\frac{\partial \sigma\left[\varepsilon^{(i)}(z, y)\right]}{\partial \varepsilon} \rightarrow E_{T}\left(\eta^{(i)}\right)=\frac{\partial \sigma\left[\varepsilon\left(\eta^{(i)}\right)\right]}{\partial \varepsilon} .
\end{aligned}
$$

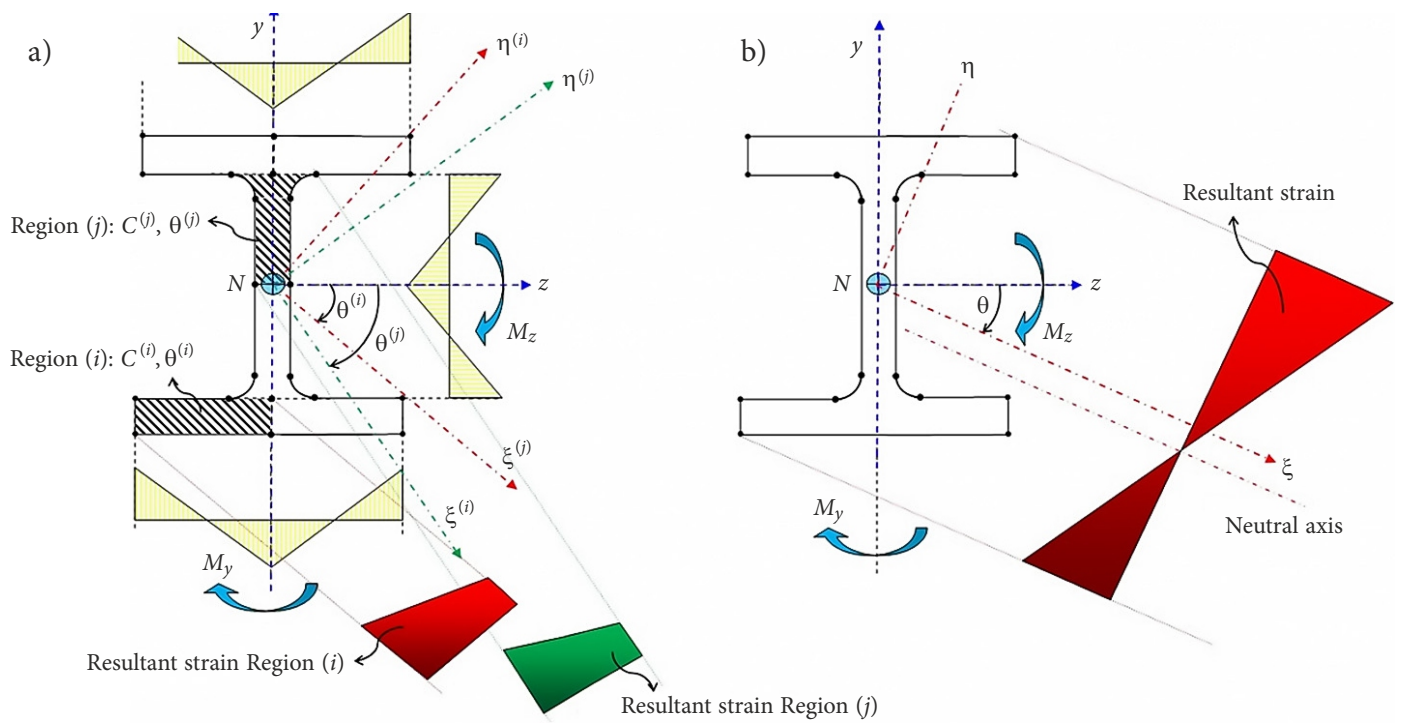

Fig. 5. Uniform stress distribution for encased steel profile. Rotation of the reference axes: (a) with residual stresses; (b) without residual stresses 
As already mentioned the steel cross-section is conceptually decomposed in a specific number of regions $(n C)$, with distinct angle of rotation $\theta^{(i)}$, and defined as a counter-clockwise series of $(n)$ vertex coordinates $(z, y)$ and by the radius $r$ of the succeeding circular arc segment (ignored for straight segments). Hence, the resultant actions of the steel component can be obtained as the summation of individual region contributions, by applying the Green's theorem on each region separately, as follows:

$$
\begin{aligned}
& I=\iint_{A} \sigma^{(r)}[\varepsilon(z, y)] z^{m} y^{n} d z d y= \\
& \sum_{i=1}^{n C} \oint_{C^{(i)}} \sigma^{(r)}\left[\varepsilon\left(\eta^{(i)}\right)\right] \mathrm{H}\left(\xi^{(i)}, \eta^{(i)}\right) d \eta^{(i)}=\sum_{i=1}^{n C} I\left(\theta^{(i)}\right),
\end{aligned}
$$

where the surface boundary $C^{(i)}$ is represented by a closed polygon composed of straight segments and circular arches defined by the vertex coordinates $\left(\xi_{j}^{(i)}, \eta_{j}^{(i)}\right)$ and radius $r_{j}^{(i)}$ (Chiorean 2017):

$C^{(i)} \rightarrow\left[\left(\xi_{1}^{(i)}, \eta_{1}^{(i)}, r_{1}^{(i)}\right), \quad\left(\xi_{2}^{(i)}, \eta_{2}^{(i)}, r_{2}^{(i)}\right), \ldots,\left(\xi_{n}^{(i)}, \eta_{n}^{(i)}, r_{n}^{(i)}\right)\right]$.

By decomposing the integral over the perimeter $C_{i}\left(I\left(\theta^{(i)}\right)\right)$ for each individual segment and circular arch $(j)$ along the perimeter $\left(C_{i}\right)$ the line integral $I\left(\theta^{(i)}\right)$ can be further simplified into a line integral of a single-variable function $F\left(\eta^{(i)}\right)$ as:

$$
\begin{aligned}
& I\left(\theta^{(i)}\right)=\oint_{C^{(i)}} \sigma^{(r)}\left[\varepsilon\left(\eta^{(i)}\right)\right] \mathrm{H}\left(l\left(\eta^{(i)}\right), \eta^{(i)}\right) d \eta^{(i)}= \\
& \oint_{C^{(i)}} F\left(\eta^{(i)}\right) d \eta^{(i)}=\sum_{j=1}^{n l_{i}} \int_{\eta_{j}^{(i)}}^{\eta_{j+1}^{(i)}} F\left(\eta^{(i)}\right) d \eta^{(i)},
\end{aligned}
$$

where $n l_{i}$ denotes the number of segments and circular arches of the perimeter $\left(C_{i}\right)$. Taken into account the independence of parametrization of the curve and the fact that for line integrals, when adding two regions with a common edge the common edges are traversed in opposite directions, the line integrals given in Eq. (31) have to be computed only on the outside boundary as is shown in Figure 6. Hence the integral $I$ given in Eq. (31) is evaluated only on the positive oriented boundary of cross-section denoted here as $\partial A$.

The boundary of steel cross-section is divided in distinct $(n L)$ edges (i.e. straight lines and circular arcs) according with the geometry of the steel cross-section (Fig. 6) such that (Chiorean 2017):

$$
\partial A=\sum_{k=1}^{n L} \partial A^{k} .
$$

Thus the line integrals are evaluated for each sides as follows (Fig. 6):

$$
I=\sum_{i=1}^{n C} \oint_{C^{(i)}} F\left(\eta^{(i)}\right) d \eta^{(i)}=\sum_{k=1}^{n L} \oint_{\partial A^{(k)}} F\left(\eta^{(k)}\right) d \eta^{(k)}
$$

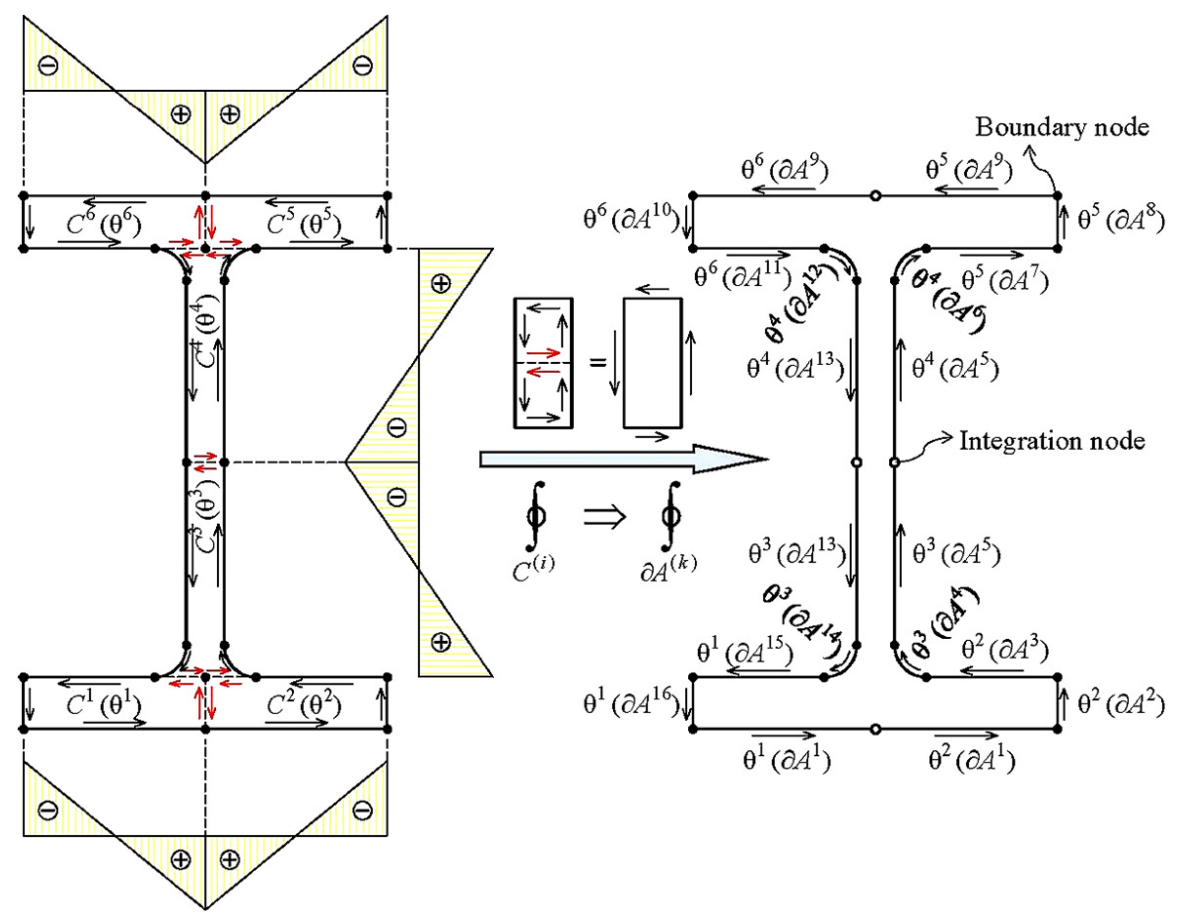

Fig. 6. Integration on the boundary $\partial A$ of the steel cross-section 
In this way the difficulties of integration on separate regions over entire steel cross-section may be avoided and such an approach leads to significant saving in computational effort and in inputting the data to describe the cross-section shapes, and allows also efficiently handling various circular shapes such as fillet regions (Fig. 3) which define the exact geometry of the encased structural steel profiles.

For hollow cross-sections we define two types of cross-section boundaries, the external one, whose contribution is added to the section response and the internal one respectively, whose contribution is subtracted from the cross-section response, allowing in this way the efficient embedment of voids. In order to identify the various regions in a complex composite steel-concrete cross-section with different material properties (e.g. concrete and structural steel) each region (component) with assigned material properties is treated separately (Charalampakis, Koumousis 2008). The conventional steel reinforcements are assumed to be discrete points with effective area, co-ordinates and stresses and to avoid double counting of the concrete area that is displaced by the steel bars, the concrete stress at the centroid of the steel bars is subtracted from the reinforcement bar force (Charalampakis, Koumousis 2008).

In order to perform the integral on a determined side of the contour $\left(\partial A_{k}\right)$ of the integration area, an interpolatory Gauss-Lobatto method is used. In the case of the steel profile, for edges in which residual stresses changes their variation, the intergation rule is applied by dividing the interval of integration into two sub-intervals, according with changes in the definition of the residual stress distribution, and use the quadrature rule for each sub-interval (Fig. 5). Since the point corresponding to the left end in one interval is the same as the point corresponding to right end in the next and assuming that we keep the values of the integrand at the interval endpoints after we have evaluated them and reuse where appropriate, the cost of evaluating a Lobatto rule is reduced by about one integrand evaluation compared with Legendre rule (Chiorean 2010).

It is important to highlight that when the stress field is defined by a piecewise function (e.g. elasticperfect plastic or by combination of different functions for ascending part and descending part respectively as assumed usually for concrete in compression) and there is no continuity in the derivative (i.e. weak discontinuity), the interpolatory integration methods can produce important integration errors (Bonet et al. 2004). In such situations an adaptive strain-mapping quadrature strategy as suggested in (Papanikolau 2012) may be applied in conjunction with the Gauss-Lobatto integration rule for each function part within each side by dividing the interval of integration according with changes in the definition of the stress function, and then use the adaptive quadrature rule in each subinterval (Chiorean 2010).

\section{Computational examples}

\subsection{Composite steel-concrete cross-section with arbitrary shape}

The composite steel-concrete cross-section depicted in Figure 7 (Chen et al. 2001) consists of the concrete matrix, fifteen reinforcement bars of diameter $18 \mathrm{~mm}$, a structural steel element and a circular opening. Characteristic strengths for concrete, structural steel and reinforcement bars are $f^{\prime \prime}{ }_{c}=30 \mathrm{Mpa}, f_{s t}=355 \mathrm{Mpa}$ and $f_{s}=460 \mathrm{Mpa}$, respectively. These characteristic strengths are reduced by dividing them with the corresponding safety factors $\gamma_{c}=1.50, \gamma_{s t}=1.10$ and $\gamma_{s}=1.15$. The stress-strain curve for concrete which consists of a parabolic and linear-part was used in the calculation, with the crushing strain $\varepsilon_{0}=0.002$ and ultimate strain $\varepsilon_{c u}=0.0035$. The Young modulus for all steel sections was $200 \mathrm{GPa}$ while the maximum strain was $\varepsilon_{u}= \pm 1 \%$. A bi-linear elasto-perfect plastic stressstrain relationship for the reinforcement bars and structural steel, both in tension and in compression, is assumed.

The strain softening effect for the concrete in compression is taken into account, in the present ap-

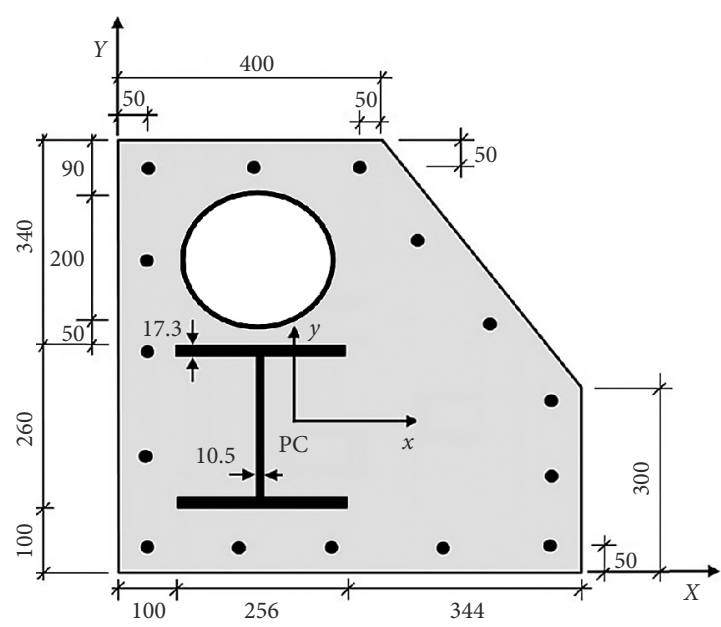

Fig. 7. Composite steel-concrete cross-section 
Table 2. Main parameters involved in the iterative process $\left(N=6000 \mathrm{kN}\right.$; reference strain $\left.\varepsilon_{c}=0.004 ; \alpha=15^{\circ}\right)$

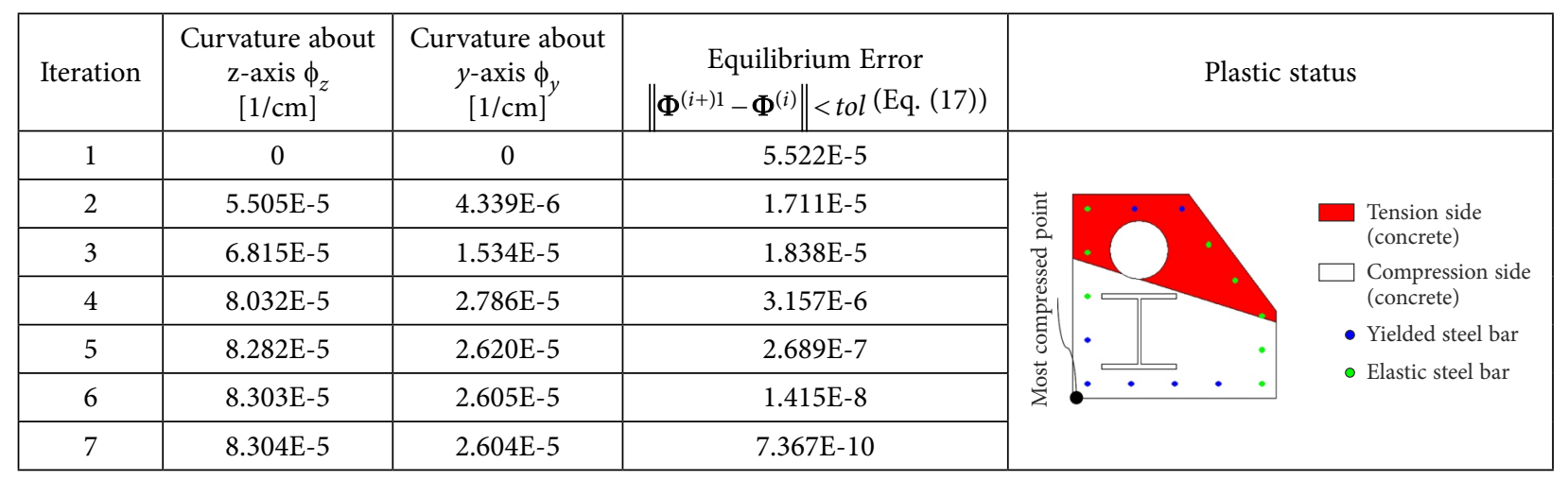

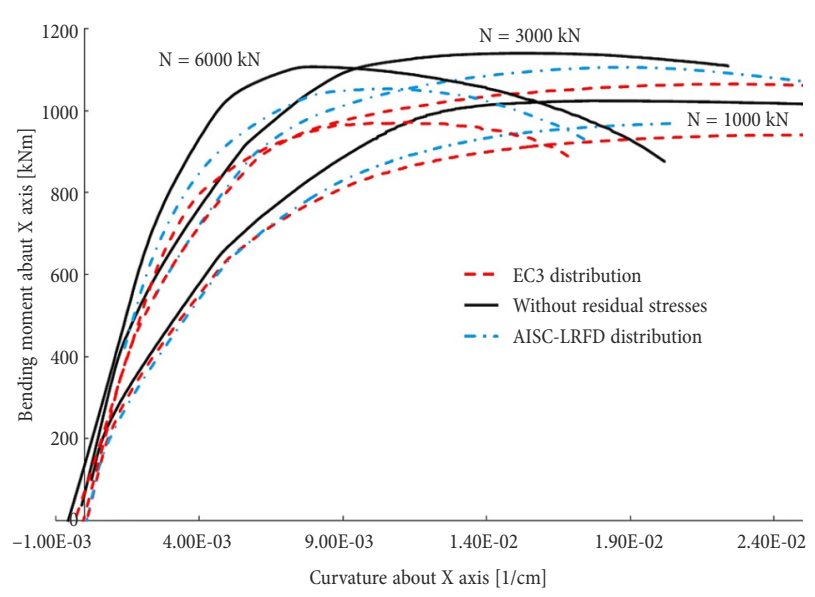

Fig. 9. Moment curvature diagrams for different values of compressive axial force: Influence of residual stresses

Two distributions according with EC3 and AISC-LRFD respectively of the residual stresses have been considered for the encased steel profile. As it can be seen the presence of residual stresses reduces significantly the ultimate strength capacity of cross-section and also the flexibility of the cross-section increasing significantly in the presence of residual stresses and this effect becomes more explicit with highest values of axial load.

\subsection{Rectangular composite steel-concrete section. Influence of residual stresses and geometry.}

In order to evaluate the effects of residual stresses and precise modelling of the geometry of encased steel profile over ultimate strength capacity and deformability of composite steel-concrete cross section, the moment-curvature diagrams are determined for a rectangular cross-section with symmetrically placed structural steel. The cross-section consists of a concrete core $(40 \times 40 \mathrm{~cm})$ and a symmetrically placed $\mathrm{Eu}-$ ropean section HEB 260 (Fig. 10) and four reinforced

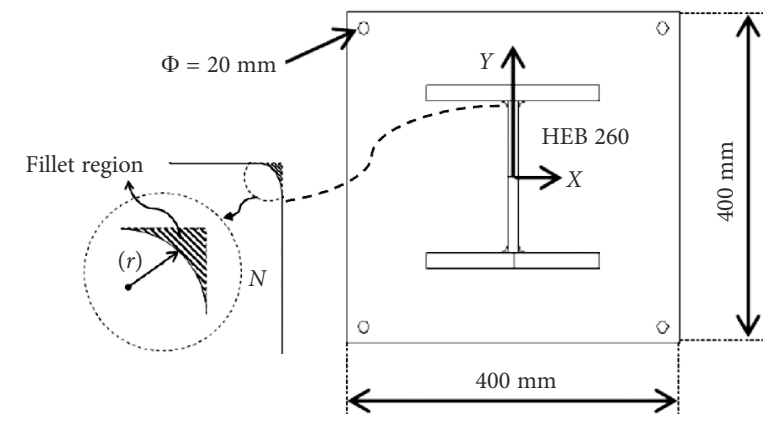

Fig. 10. Rectangular composite steel-concrete cross-section

bars with $20 \mathrm{~mm}$ diameter. Characteristic strength for concrete in compression is ${f^{\prime \prime}}_{c}=20 \mathrm{MPa}$ and the stress-strain curve given by the Eq. (2) is considered, with crushing strain $\varepsilon_{0}=0.002$ and $\varepsilon_{\mathrm{cu}}=0.0035$ and $\gamma=0.15$. A bi-linear elasto-perfect plastic stress-strain relationship for the reinforcement bars and structural steel, both in tension and in compression, is assumed with the yield strength $f_{y}=300 \mathrm{MPa}$ and the Young modulus is $200 \mathrm{GPa}$. This example has been studied previously in (Chiorean 2013) where the explicit solution of the non-linear equilibrium Eqs (5) is solved in conjunction with an arc-length iterative procedure. Figures 11-13 reports the comparative bending moment-curvature diagrams of cross-section, considering uniaxial bending about $X$-axis (strong) and $Y$ axis (weak) respectively and bi-axial bending (Fig. 13) under several compressive axial loads. No significant differences have been obtained between the solutions given in (Chiorean 2013) and those obtained with the proposed approach, therefore these comparative results are not depicted in Figure 1 and Figure 12 respectively. The effect of residual stresses is taken into account considering two types of residual stress patterns, EC3 and AISC-LRFD, respectively, for structural steel element. These comparisons reveal that the pres- 
ence of residual stresses play an important role on both carrying capacity and inelastic behaviour, during the loading process, and this effect becomes more effective with highest compressive axial load and when bend-

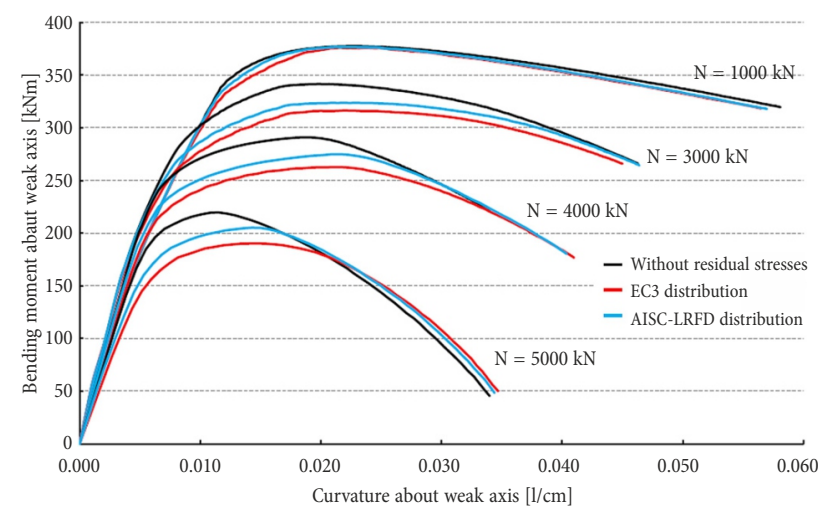

Fig. 11. Moment-curvature analysis for different values of compressive axial loads with and without residual stress effects: weak axis bending

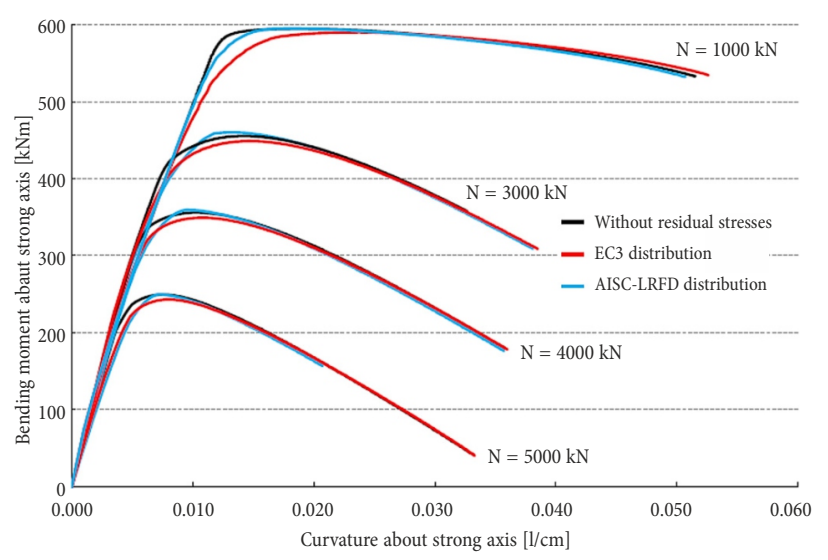

Fig. 12. Moment-curvature analysis for different values of compressive axial loads with and without residual stress effects: strong axis bending

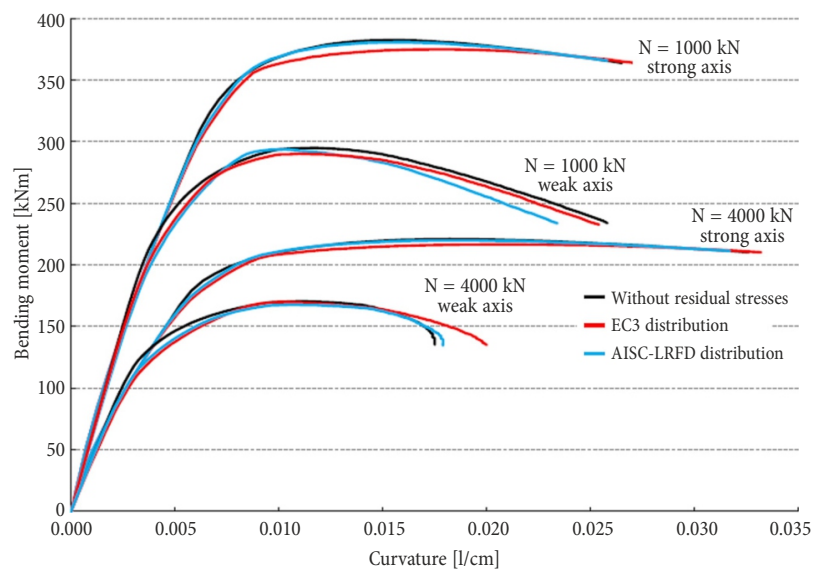

Fig. 13. Moment-curvature analysis for different values of compressive axial loads with and without residual stress effects: Bi-axial bending $\alpha=30^{\circ}$ ing takes place about weak axis bending. The effect of shape geometry of cross-section, including or excluding the fillet regions in definition of the section geometry, over inelastic behaviour and ultimate strength capacity has been also investigated in Figure 14. As it can be seen neglecting the fillet regions indicates lower stiffness and lower strength capacity of cross-section, even for low values of axial force, underestimating the ultimate strength capacity of cross-section until $10 \%$ as compared with the cases when the fillet regions are modelled. Consequently, the exact modelling of geometry of encased steel profile must be considered in a valuable advanced inelastic analysis method.

\subsection{Reinforced concrete box cross-section}

The box cross-section, depicted in Figure 15, consists of the concrete matrix and sixteen reinforcement bars, all rebars having the same diameter (RodriguezGutierrez, Dario Aristizabal-Ochoa 2001). Characteristic strengths for concrete and reinforcement bars are: $f_{c}^{\prime \prime}=23.443 \mathrm{MPa}, f_{y}=413.69 \mathrm{MPa}$ respectively.

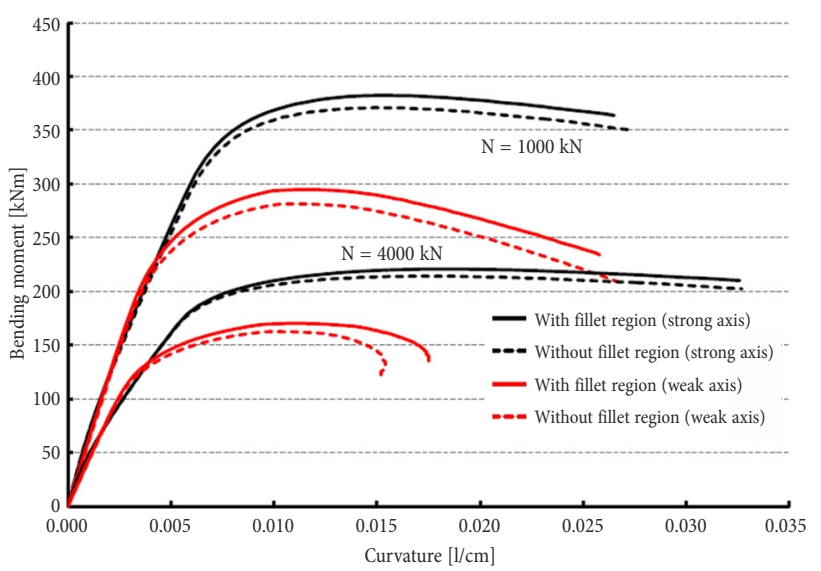

Fig. 14. Moment-curvature analysis for different values of compressive axial loads with and without considering the fillet region effects: $\mathrm{Bi}$-axial bending $\alpha=30^{\circ}$

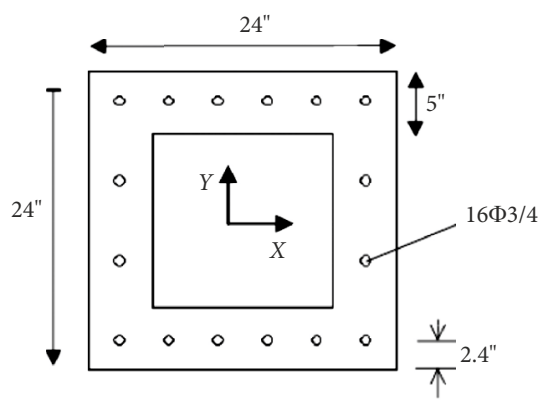

Fig. 15. Biaxially loaded box cross-section 
The stress-strain curve for concrete in compression given by the Eq. (2) was used in the calculation, with the crushing strain $\varepsilon_{0}=0.002$ and ultimate strain $\varepsilon_{\mathrm{cu}}=0.0035$. A bi-linear elasto-perfect plastic stressstrain relationship for the reinforcement bars, both in tension and in compression, is assumed, the Young modulus for reinforcing bars was $200 \mathrm{GPa}$ while the maximum strain was $\varepsilon_{u}= \pm 1 \%$. The strain softening effect for the concrete in compression is taken into account, through the parameter $\gamma$.

Figures 16 and 17 show the moment-curvature diagrams of cross-section, considering bi-axial bending $\left(\alpha=26.56^{\circ}\right)$ for a compressive axial force $N=6000 \mathrm{kN}$ and for different values of degree of

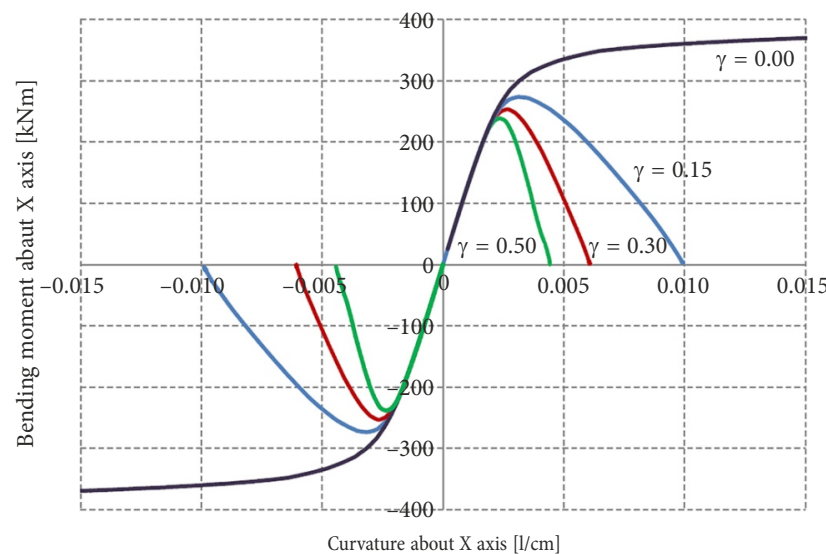

Fig. 16. Moment curvature diagrams for different values of degree of confinement and compressive axial force of $N=6000 \mathrm{kN}$ and $\alpha=26.56^{\circ}$ : Bending moment about $X$-axis (strong axis) confinement $(\gamma=0 ; 0.15 ; 0.30 ; 0.50)$. As it can be seen, the strain softening effect exhibited by the concrete in compression $(\gamma>0)$ indicates lower bending capacity of cross section and also "snap-back" behaviour for bending-moment-curvature about weak axis; this effect is more accentuated as degree in confinement is decreased. This example proves the efficiency of the numerical procedure developed here to determine the complete moment-curvature relationship even for a very sharp descendent branch for concrete in compression $(\gamma=0.50)$. No convergence difficulties have been encountered during the loading process, usually the equilibrium has been achieved within four to five iterations with an equilibrium tolerance tol $=1 \mathrm{E}-10$.

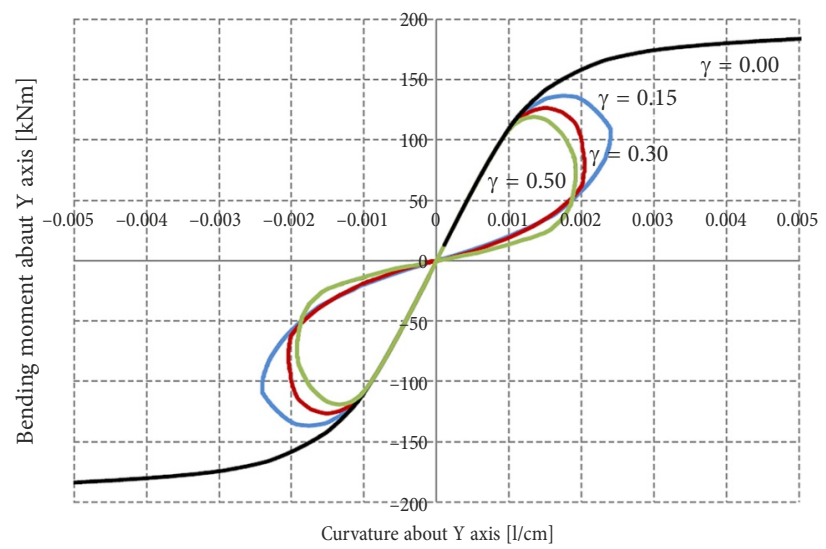

Fig. 17. Moment curvature diagrams for different values of degree of confinement and compressive axial force of $N=6000 \mathrm{kN}$ and $\alpha=26.56^{\circ}$ : Bending moment about $Y$-axis(weak axis)

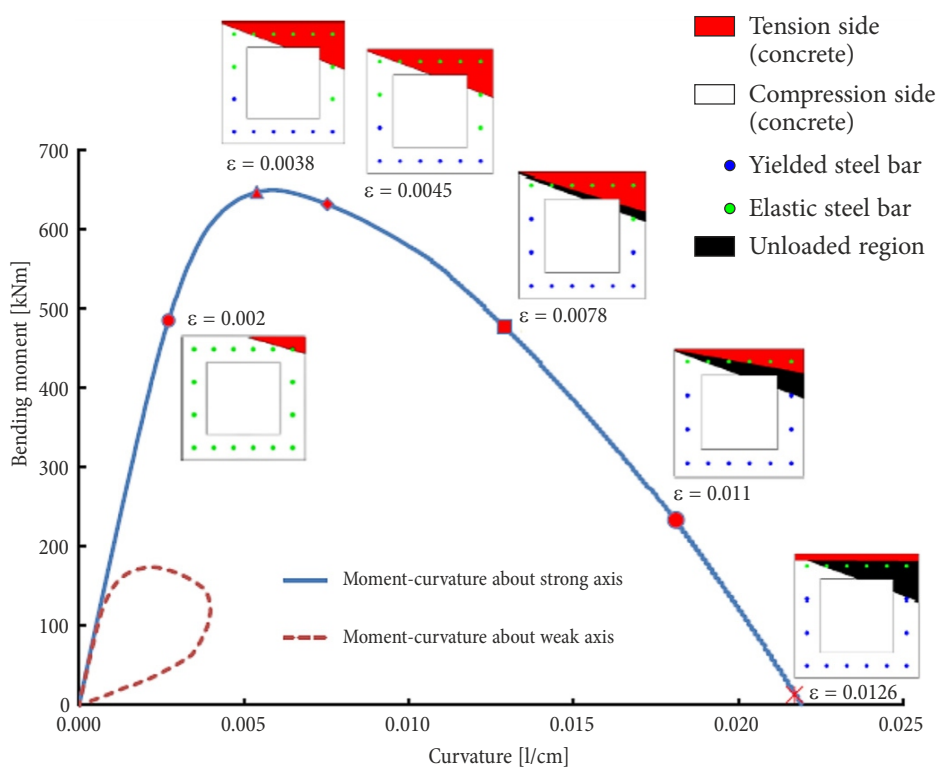

Fig. 18. Complete moment curvature diagram for axial force of $N=6000 \mathrm{kN}$ and $\alpha=15^{\circ}$ : Plastic status of cross-section for different values of maximum compressive strain 
Figure 18 shows a detailed analysis concerning the inelastic behaviour (spread of plasticity) throughout the cross-section when the section is subjected to bi-axial bending $\left(\alpha=15^{\circ}\right)$ and compressive axial force $\mathrm{N}=6000 \mathrm{kN}$ and for the strain-softening parameter $\gamma=0.15$. The complete moment-curvatures have been depicted both for strong and weak axis of bending and the plastic status of the cross-section associated to different values of the reference strain imposed in the most compressed point of the cross section has been revealed on the graph. As it can be seen from the compressive strain of 0.0045 unloading (dark regions) of some regions of the cross-section starts to begin despite the fact that the compressive strain in most compressed point is continously increased. This computional example highlits that even for a monotonically increasing of the reference strain some regions of the cross-section may unloads when strain softening for the concrete in compression is taken into account, hence the necessity to implement a stress-path dependency for an more accurate inelastic analysis of cross-sections.

\section{Conclusions}

A new computer method has been developed for moment-curvature analysis of composite steel-concrete cross-sections subjected to axial force and biaxial bending moments. The main feature of the proposed approach consists in controlling the nonlinear inelastic response at the strains level enforcing in the same time the elato-pastic equilibrium for a prescribed axial force and bending moments ratio. Such an approach allows a very precise evaluation of the strain and stress state throughout the arbitrary shaped cross-section allowing direct evaluation of the inelastic curvatures from a prescribed value of the strain in the most compressed concrete fibre. In the proposed approach the nonlinear equilibrium equations associated to axial force and bending moments are manipulated so that one of them is uncoupled and the Newton iterative strategy is applied only to the remaining coupled equilibrium equations. From the extensive numerical experiments, some of them presented in this work, has been found that the convergence stability is not sensitive to the initial/starting values of the iterative process and to the strain softening exhibited by the concrete in compression since the Jacobian's of the resulted nonlinear system of equations is always positive definite. In the pro- posed approach an improved adaptive Gauss-Lobatto numerical integration scheme on a Green path integral is applied demonstrating fast execution and accuracy for stress integration throughout the cross-sections. A particularly important feature of the proposed path integral approach is represented by the ability to model the residual stresses in the case of encased steel-profile without the need to decompose the steel component in distinct regions according with changes in the definition of the residual stress distribution.

Using the proposed approach we have found that the strain softening effect exhibited by the concrete in compression indicates lower bending capacity of cross section and also "snap-back" behaviour of the momentcurvature response; this effects are more accentuated as degree in confinement is decreased. The numerical studies carried out in the present study reveals that the presence of the residual stresses, for encased steel section, play an important role on both ultimate strength capacity and inelastic behaviour, at different stages of loading, and this effect becomes more effective with highest compressive axial load especially when bending takes place about weak axis of the steel profile. The effect of the fillet regions in definition of the section geometry of the encased steel profile over inelastic behaviour and ultimate strength capacity has been also investigated in this work and can be concluded that the exact modelling of geometry of cross-section must be considered in a valuable advanced analysis method since the neglecting the fillet regions may lead to an underestimation of the ultimate strength capacity and deformability of the composite cross-sections.

Furthermore, the numerical study conducted by the proposed approach reveals that in order to determine the complete moment-curvature diagram (i.e. post-critical response) for a constant bending moments ratio a stress-path dependency must be taken into account because unloading regions may develop in the presence of the strain-softening effect exhibited by the concrete in compression even when the strain in the most compressed point is progressively increased.

It can be concluded that the proposed numerical method proves to be reliable and accurate for practical applications in the design of composite steel-concrete cross-sections and can be implemented in the advanced nonlinear inelasic analysis techniques of $3 \mathrm{D}$ composite frame structures.

Since the solution of the nonlinear equilibrium equations is controlled by the predefined values of 
strain in most compressed concrete fiber, this approach seems to be effective to be applied for a more complex cyclic moment-curvature analyses, by continuously varying (increasing-loading/decreasing-unloading) the extreme concrete fiber strain, and in this way the complete hysteretic moment-curvature diagram for given bending moment's ratio at prescribed axial force can be revealed.

Future studies are envisaged concerning the extension of the proposed approach to cyclic momentcurvature analysis of arbitrary shaped composite steelconcrete cross-sections.

\section{References}

Bonet, J. L.; Romero, M. L.; Miguel, P. F.; Fernandez, M. A. 2004. A fast stress integration algorithm for reinforced concrete sections with axial loads and biaxial bending, Computers and Structures 82: 213-225.

https://doi.org/10.1016/j.compstruc.2003.10.009

CEB-FIP 1993. Model Code 1990: Design Code. Comité Euro-International du Béton. Thomas Thelford, London, UK.

Charalampakis, A. E.; Koumousis, V. K. 2008. Ultimate strength analysis of composite sections under biaxial bending and axial load, Advances in Engineering Software 39(11): 923936. https://doi.org/10.1016/j.advengsoft.2008.01.007

Chen, S. F.; Teng, J. G.; Chan, S. L. 2001. Design of biaxially loaded short composite columns of arbitrary section, Journal of Structural Engineering, ASCE 127(6): 678-85. https://doi.org/10.1061/(ASCE)0733-9445(2001)127:6(678)

Chiorean, C. G. 2010. Computerised interaction diagrams and moment capacity contours for composite steel-concrete cross-sections, Engineering Structures 32(11): 3734-3757. https://doi.org/10.1016/j.engstruct.2010.08.019
Chiorean, C. G. 2013. A computer method for nonlinear inelastic analysis of 3D composite steel-concrete frame structures, Engineering Structure 57: 125-152.

https://doi.org/10.1016/j.engstruct.2013.09.025

Chiorean, C. G. 2014. Moment-curvature analysis of composite steel-concrete cross-sections of arbitrary shape, in Proceedings of the Twelfth International Conference on Computational Structures Technology, 2-5 September 2014, Neaples, Italy.

Chiorean, C. G. 2017. Second-order flexibility-based model for nonlinear inelastic analysis of 3D semi-rigid steel frameworks, Engineering Structures 136: 547-579. https://doi.org/10.1016/j.engstruct.2017.01.040

Marmo, F. 2007. A fiber-free approach to the inelastic analysis of reinforced concrete structures: $\mathrm{PhD}$ Thesis. University Napoli „Federico II".

Marmo, F.; Rosati, L.; Serpieri, R. 2008. Influence of prestressing and softening on the ultimate strength domains of RC sections subject to axial force and biaxial bending, in Proceedings of Advances in Reinforced Concrete and Precast Constructions, 5-6 December 2008, Brescia, Italy.

Papanikolau, V. K. 2012. Analysis of arbitrary composite sections in biaxial bending and axial load, Computers and Structures 98-99: 33-54. https://doi.org/10.1016/j.compstruc.2012.02.004

Rodriguez-Gutierrez, J. A.; Dario Aristizabal-Ochoa, J. 2001. M-P- $\Phi$ diagrams for reinforced, partially, and fully prestressed concrete sections under biaxial bending and axial load, Journal of Structural Engineering, ASCE 127(7): 763-773. https://doi.org/10.1061/(ASCE)0733-9445(2001)127:7(763)

Rotter, J. M. 1985. Rapid exact inelastic biaxial bending analysis, Journal of Structural Engineering, ASCE 111(12): 2659-67. https://doi.org/10.1061/(ASCE)0733-9445(1985)111:12(2659)

Vecchio, F. J.; Collins, M. P. 1986. The modified compression field theory for reinforced concrete elements subjected to shear, ACI Structural Journal 83: 219-231.

Cosmin G. CHIOREAN is Professor of Structural Engineering at the Technical University of Cluj-Napoca (TUCN), Faculty of Civil Engineering, Romania. He graduated Faculty of Civil Engineering from TUCN (Civil Engineering section) in 1995 and also Mathematics and Computer Science from Babes-Bolyai in 1999. In 2001 he got his PhD in Structural Engineering from Technical University of Cluj-Napoca, Romania. He spent two years as a postdoctoral researcher at New University of Lisbon, Faculty of Science and Technology, Lisbon, Portugal. Since 2013 Prof. Chiorean is PhD supervisor in the field of Civil Engineering, he received his habilitation in civil engineering from Technical Univ. of Construction, Bucharest, Romania. Prof. Chiorean's research interests focus on improving the computational methods for design of civil structures, industrial buildings and bridges. His research includes both computational and experimental techniques with emphasis on the development and application of advanced numerical methods to solve problems relevant to structural and geotechnical engineering. 